\title{
Reliable Hopping Sequence Design for Highly Interfered Wireless Sensor Networks
}

\author{
Samuele Zoppi, H. Murat Gürsu, Mikhail Vilgelm, Wolfgang Kellerer \\ Chair of Communication Networks \\ Technical University of Munich, Germany \\ Email:\{samuele.zoppi, murat.guersu, mikhail.vilgelm, wolfgang.kellerer\}@tum.de
}

\begin{abstract}
Guaranteeing reliability in highly interfered environments is a challenging requirement of current and future wireless applications. A promising state-of-the-art solution for low-power wireless technologies, e.g., wireless sensor networks (WSNs), is frequency hopping aided with black- and white-listing of channels. Both methods, although increase reliability, sacrifice frequency resources. Extensive measurements of channels' packet drop probabilities show that interfered channels are not fully blocked. Motivated by this discovery, we propose the whitening - a methodology for reliable hopping sequence design without resource sacrifice. We model the efficiency of interfered ISM band channels, and study the gains and trade-offs of applying whitening in different scenarios. Application reliability is achieved by granting re-transmissions within a time deadline. Simulations and measurements, performed on the exemplary use case of Time Slotted Channel Hopping WSNs, show that the proposed methods outperform state-of-the-art solutions in the presence of interference in terms of reliability.
\end{abstract}

\section{INTRODUCTION}

In the last decade, we experienced a considerable increase of wireless applications, resulting in a huge demand of wireless resources. A clear example of this reality is the overload condition of the $2.4 \mathrm{GHz}$ unlicensed band. Several technologies like Wi-Fi, Bluetooth, Wireless Sensor Network (WSN) are now sharing the same spectrum, and in the future it is foreseen that additional technologies will join this band as well, for instance, LTE-Unlicensed [1].

From the perspective of delay-constrained reliable applications, the coexistence of several technologies in the same spectrum creates a serious issue [2]. One particular example for coexistence is the future on-board aircraft communication, where wires will be eliminated in favor of wireless links. A complete future avionic system is foreseen to deploy both WiFi and WSN over the same wireless resources [2]. The former is used to deliver entertainment application, and the latter to replace sensors and actuators information. This will result in lighter and more flexible airplane cabins, with the requirement of guaranteeing hard reliability and delay constraints. However, as shown in [3], the presence of Wi-Fi interference severely degrades the WSN performances.

A well established technique for WSN to deal with highly interfered environments is channel hopping, which consists of selecting different frequencies between subsequent transmissions, exploiting frequency diversity and aiming at decreasing correlation between packet drops.

\section{A. Related Work}

A number of studies has been dedicated to channel hopping so far. Its impact on link connectivity and routing in a meshed network has been discussed in [4]. The authors show the benefits of deploying channel hopping and discuss whitelisting - reducing the hopping sequence to non-interfered channels. Also several experimental studies of Time Slotted Channel Hopping (TSCH) [5] in WSN have been conducted. The main advantage of TSCH with respect to the IEEE 802.15.4 standard is the combination of TDMA structure with frequency hopping. Adopting TSCH, a comparison with adaptive singlechannel routing techniques in [6] has shown superiority of channel hopping in sparse networks. In [7], the authors have compared scheduled and random access-based MAC for multihop TSCH, and have shown that they deliver similar reliability for low-load networks. While these studies provide insights into the benefits of channel hopping, they do not address design of the hopping sequence.

The state-of-the-art technique of optimizing channel hopping is blacklisting - excluding highly interfered channels from the hopping sequence. Adaptive TSCH has been introduced in [8], and it extends TSCH with dynamic blacklisting. The idea was developed further as Enhanced TSCH in [9]. The practical hardware aspects of hopping sequence generation were addressed in [10]. Although several protocols in WSN propose the assignment of multiple channels for communications [11], none of the state-of-the-art solutions address the issue that blacklisting and whitelisting are shrinking the number of available resources for parallel communication.

\section{B. Contributions of the Paper}

In this paper, we focus on designing reliable hopping sequence without sacrificing resources. In our previous work [12], we noticed from experimental results that interfered channels are not fully blocked, and non-interfered channels are affected by small, but not negligible, packet drops. This consideration naturally raised the following questions: What is the capacity of interfered channels? Which gains can we have in terms of reliability and latency? In this research, we answer these questions bringing the following contributions: (i) modeling of resource block success probability for $\mathrm{TSCH}$, gains of the proposed hopping sequence design with respect to whitelisting; (ii) introduction of whitening hopping sequence design, a technique aimed at increasing reliability without 
sacrificing resources. (iii) Extensive simulations in several interference conditions, and indoor measurements.

The remainder of this paper is structured as follows, Section II describes our scenario where WSNs are highly interfered by Wi-Fi. Section III-A provides the model of resource block success probability for TSCH. Section III-B describes the design of a whitening-based hopping sequence, Section IV presents the benefits of our hopping strategies compared to the state-of-the-art solutions. Finally, Section V concludes this paper with a summary.

\section{AN OVERVIEW OF WSNS INTERFERED BY WI-FI}

This Section presents the channel model and the wireless technologies used for the simulation and measurements. Our scenario considers future on-board aircraft communication where Wi-Fi and several WSNs physically coexist delivering entertainment, and sensors and actuators information.

In the paper, we refer to the standard IEEE 802.15.4 WSN channels as channels, and to the standard IEEE 802.11 Wi-Fi channels as Wi-Fi channels.

\section{A. Channel Model}

In order to simulate the environment described in the previous Section, we adopt a specific state-of-the-art wireless channel model for WSNs. As shown in [13], every transmission experiences a channel realization obtained by adding path loss and multi-path fading. The received power for a transmission is

$$
P_{\mathrm{RX}}=P_{\mathrm{TX}} \cdot P_{\mathrm{PL}} \cdot P_{\mathrm{FF}},
$$

where $P_{\mathrm{TX}}$ denotes the transmission power, $P_{\mathrm{FF}}$ and $P_{\mathrm{PL}}$ capture the amount of reflection and attenuation respectively of a particular environment,

$$
P_{\mathrm{PL}}=\left(\frac{c}{4 \pi f d}\right)^{\eta} \quad P_{\mathrm{FF}} \sim \ln \mathcal{N}\left(0, \sigma^{2}\right) .
$$

The path loss $P_{\mathrm{PL}}$ depends on the receiver distance $d$, carrier frequency $f$, speed of light $c$ and the path loss exponent $\eta$. Multi-path $P_{\mathrm{FF}}$ is modeled with a log-normal distribution, suitable for short-range indoor propagation [13] which depends on $\sigma^{2}$, the amount of multi-path fading in the environment.

In this paper, the values of $\eta$ and $\sigma^{2}$ are set to 2.23 and 6.2 respectively. As shown in [14], these are suitable values for WSN hardware with CC2420 radio, which are used by us for the measurements.

\section{B. IEEE 802.15.4 Physical Layer}

WSNs adopt the IEEE 802.15.4 physical layer for transmission. The simulator follows the exact same model for packet detection after propagation and interference. For every packet, the resulting Signal-to-Interference-and-Noise ratio:

$$
\gamma=\frac{P_{\mathrm{RX}}^{\mathrm{S}}}{P_{\mathrm{N}}+\sum_{i=0}^{N_{\mathrm{IF}}} P_{\mathrm{RX}}^{\mathrm{IF}_{i}}},
$$

where $P_{\mathrm{N}}$ is the noise power floor, $N_{\mathrm{IF}}$ is the number of received interfering signals; $P_{\mathrm{RX}}^{\mathrm{S}}, P_{\mathrm{RX}}^{\mathrm{IF}}$ are the received powers of signal and interference, respectively.
As shown in [13], Eq. (3) can be used to calculate the values of $\frac{E_{b}}{N_{0}}$, Bit Error Rate (BER) and consequent Packet Error Rate (PER) for a received packet using the 802.15.4 physical layer. A generic O-QPSK modulation with Direct-Sequence Spread Spectrum (DSSS) has

$$
\mathrm{BER}=\mathcal{Q}\left(\sqrt{\frac{2 M \frac{B_{N}}{R} \gamma}{M+\frac{4 B_{N}}{3 R}\left(N_{\mathrm{U}}-1\right) \gamma}}\right),
$$

where $M$ is the number of chips per bit; $N_{U}$ the number of simultaneously transmitting users; $B_{N}$ is the bandwidth of the noise; $R$ is the data rate of the communication.

The resulting PER for a packet of length $l$ is:

$$
\mathrm{PER}=1-(1-\mathrm{BER})^{l} \text {. }
$$

Finally, in the simulator, the result of Eq. (5) is fed in a Bernoulli distribution that simulates the successful reception of the packet.

\section{IEEE 802.15.4e MAC}

Our WSN devices adopt the IEEE 802.15.4e channel hopping mechanism, also denoted as TSCH. The main advantage of TSCH with respect to the legacy 802.15.4 strategy is the combination of TDMA structure with frequency hopping. The synchronized time slotted access is used to guarantee deterministic delay requirements, while the continuous hopping exploits the frequency diversity to accomplish uncorrelated subsequent transmissions. Synchronization in the network is achieved by means of the Absolute Sequence Number (ASN), which indicates the global time and is broadcasted by the PAN coordinator. Time is additionally structured in cyclic frames consisting in Slot-Frame-Size (SFS) number of time slots. In the frequency domain, frequency hopping is achieved by means of a hopping sequence (HS), a lookup table containing all the available WSN channels for transmission.

The TSCH time-frequency structure enables flexible scheduling strategies. By means of a schedule, it is possible to assign different motes to frequencies and time slots available in the Slot-Frame. However, since continuous hopping is performed, the frequencies defined in the schedule are only allocated with respect to an offset from the hopping sequence,

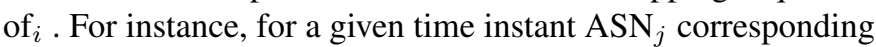
to an allocated time slot $\mathrm{ts}_{t}$ and channel offset of ${ }_{i}$, the transmission frequency is

$$
\mathrm{f}_{k}=\mathrm{HS}\left[\left(\mathrm{ASN}_{j}+\mathrm{of}_{i}\right) \bmod N_{\mathrm{CH}}\right] .
$$

For every ASN, different motes will select different offsets resulting in different allocation of channels. The hopping is performed via the modulo operation, and takes into account the total number of channels available for transmission $N_{\mathrm{CH}}$, 16 channels in the $2.4 \mathrm{GHz}$ ISM band.

\section{External Wi-Fi Interference Model}

In the model, we take external Wi-Fi interference into account. This might arise from other Wi-Fi networks, such as the entertainment system or passengers' devices in an airplane. 


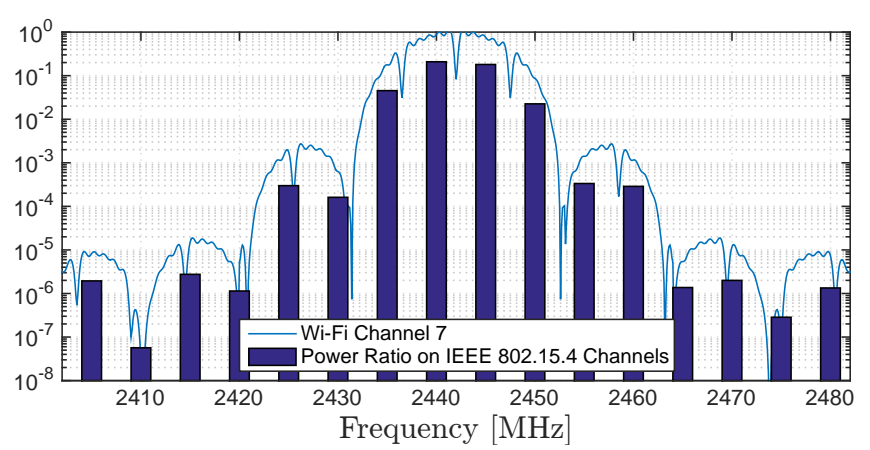

Fig. 1: Normalized Power Spectral Density (PSD) of the Wi-Fi channel 7 with power ratios on IEEE 802.15.4 channels.

Wi-Fi networks are represented as several clients connected to multiple different Access Points (APs) operating simultaneously in the $2.4 \mathrm{GHz}$ ISM band. Communication between Wi-Fi clients and APs follows the full buffer assumption, thus without interruptions. The effect is heavy usage of the ISM band with several highly interfered frequencies and few white (i.e. non interfered) frequencies.

We simulate the behavior of the Wi-Fi and we include its received power combining Eq. (1) in Eq. (3) to calculate the resulting PER with interference. The Wi-Fi interference is generated with respect to the IEEE 802.11b DSSS modulation replicating the work in [3].

Furthermore, in Fig. 1 we show the impact of interference from Wi-Fi channel 7 on the WSN channels. We can notice that the interference from Wi-Fi channel 7 manly affects WSN channels between 17 and $20(2435-2450 \mathrm{MHz})$, while Wi-Fi side lobes have a considerably lower power.

\section{Proposed Hopping Sequence Design With WHITENING APPROACH}

In the previous work [12], we measured the packet drop probability of WSN channels with high interference. Interestingly, we observed that interfered channels were not fully blocked by interference, and non-interfered channels were affected by small, but not negligible, packet drops. Motivated by this discovery, we present in this Section our findings.

First, we model the probability of success of TSCH resources, and evaluate the gains of exploiting interfered channels additionally to non-interfered ones. Then, we introduce the proposed whitening hopping sequence design, and explain how to improve reliability by using all the channels.

\section{A. Modeling the Probability of Success in TSCH}

First, we define the resource block $\mathrm{RB}_{i}$ as the $i$-th channel of a time slot in a TSCH Slot-Frame. We model its stochastic behavior with a Bernoulli distributed random variable with value $\mathrm{RB}_{i}=0$ if the transmission fails, and value $\mathrm{RB}_{i}=1$ if the transmission is successful. Then, we define its expected value as the probability of success of a resource block:

$$
\mathrm{RB}_{i} \sim \operatorname{Bern}\left(P_{\mathrm{S}_{i}}\right) \quad \mathrm{E}\left[\mathrm{RB}_{i}\right]=P_{\mathrm{S}_{i}},
$$

where $P_{\mathrm{S}_{i}}$ is the probability of success of channel $i$, and $\mathrm{E}[\cdot]$ is the expected value.

The distribution of the sum of the probabilities of success in a given time slot (TS) for all the resource blocks is

$$
\begin{aligned}
\mathrm{TS} & \sim \mathrm{RB}_{1}+\mathrm{RB}_{2}+\cdots+\mathrm{RB}_{N_{\mathrm{CH}}} \\
& =\sum_{i=1}^{N_{\mathrm{W}}} \mathrm{RB}_{i}^{\mathrm{W}}+\sum_{j=1}^{N_{\mathrm{I}}} \mathrm{RB}_{j}^{\mathrm{I}},
\end{aligned}
$$

with $\mathrm{RB}_{j}^{\mathrm{I}}, \mathrm{RB}_{i}^{\mathrm{W}}$ i.i.d random variables representing interfered and white resource blocks, and $N_{\mathrm{I}}, N_{\mathrm{W}}$ the number of interfered and white channels.

The probability of success of a time slot is:

$$
\begin{aligned}
\mathrm{E}[\mathrm{TS}] & =\sum_{i=1}^{N_{\mathrm{W}}} \mathrm{E}\left[\mathrm{RB}_{i}^{\mathrm{W}}\right]+\sum_{j=1}^{N_{\mathrm{I}}} \mathrm{E}\left[\mathrm{RB}_{j}^{\mathrm{I}}\right] \\
& =\sum_{i=1}^{N_{\mathrm{W}}} P_{\mathrm{S}_{i}}^{\mathrm{W}}+\sum_{j=1}^{N_{\mathrm{I}}} P_{\mathrm{S}_{j}}^{\mathrm{I}} .
\end{aligned}
$$

where $P_{\mathrm{S}_{j}}^{\mathrm{I}}$ and $P_{\mathrm{S}_{i}}^{\mathrm{W}}$ are the success probabilities for interfered and white channels respectively. We assume that, even though the channel loss probabilities vary, the condition $P_{\mathrm{S}_{j}}^{\mathrm{I}}<\frac{P_{\mathrm{S}_{i}}}{\alpha}(10)$ holds $\forall i, j,[12]$. The threshold $\alpha$ is the channel quality separation criterion, and accommodates small channel variations.

Finally, it is possible to calculate the success gain $\mathrm{S}_{\mathrm{G}}$ obtained using all $N_{\mathrm{CH}}$ channels against using only $N_{\mathrm{W}}$ white channels (i.e. whitelisting), that is

$$
\begin{aligned}
& \mathrm{S}_{\mathrm{G}}=\frac{\mathrm{E}[\mathrm{TS}]}{\mathrm{E}\left[\mathrm{TS}^{\mathrm{W}}\right]}=1+\frac{\mathrm{E}\left[\mathrm{TS}^{\mathrm{I}}\right]}{\mathrm{E}\left[\mathrm{TS}^{\mathrm{W}}\right]} \\
& =1+\frac{\sum_{j=1}^{N_{\mathrm{I}}} \mathrm{E}\left[\mathrm{RB}_{j}^{\mathrm{I}}\right]}{\sum_{i=1}^{N_{\mathrm{W}}} \mathrm{E}\left[\mathrm{RB}_{i}^{\mathrm{W}}\right]}=1+\frac{\sum_{j=1}^{N_{\mathrm{I}}} P_{\mathrm{S}_{j}}^{\mathrm{I}}}{\sum_{i=1}^{N_{\mathrm{W}}} P_{\mathrm{S}_{i}}^{\mathrm{W}}} .
\end{aligned}
$$

From Eq. (11) we notice that the $S_{G} \geq 1$. In particular, $\mathrm{S}_{\mathrm{G}}=1$ only when all the interfered channels are fully blocked, i.e., $P_{\mathrm{S}_{j}}^{\mathrm{I}}=0, \forall j=1, \cdots, N_{\mathrm{I}}$. Thus, as long as the interfered channels have a small, but non-zero probability of success the gain is present and $\mathrm{S}_{\mathrm{G}}>1$. The magnitude of the gain depends on the ratio between the expected gain of the interfered over the white channels.

Let us consider the scheduled access of TSCH and the IEEE 802.15.4 physical layer. We further describe $P_{\mathrm{S}_{i}}$ and BER including the details of our scenario.

$$
P_{\mathrm{S}_{i}}=1-\mathrm{PER}=(1-\mathrm{BER})^{l}
$$

The physical layer of IEEE 802.15.4 adopts the following parameters: $M=8$ chips per bit, $N_{U}=1$ transmitting user, $B_{N}=2 \mathrm{MHz}$ the bandwidth of the noise, and $R=250 \mathrm{Kbps}$ the data rate of the communication. With these values, and including Eq. (3), Eq. (4) and (12) break down to

$$
\begin{aligned}
\mathrm{BER} & =\mathcal{Q}(4 \sqrt{\gamma})=\mathcal{Q}\left(4 \sqrt{\frac{P_{\mathrm{RX}}^{\mathrm{S}}}{P_{\mathrm{N}}+\sum_{i=0}^{N_{\mathrm{IF}}} P_{\mathrm{RX}}^{\mathrm{IF}}}}\right), \\
P_{\mathrm{S}_{i}} & =1-\mathrm{PER}=(1-\mathcal{Q}(4 \sqrt{\gamma}))^{l} .
\end{aligned}
$$




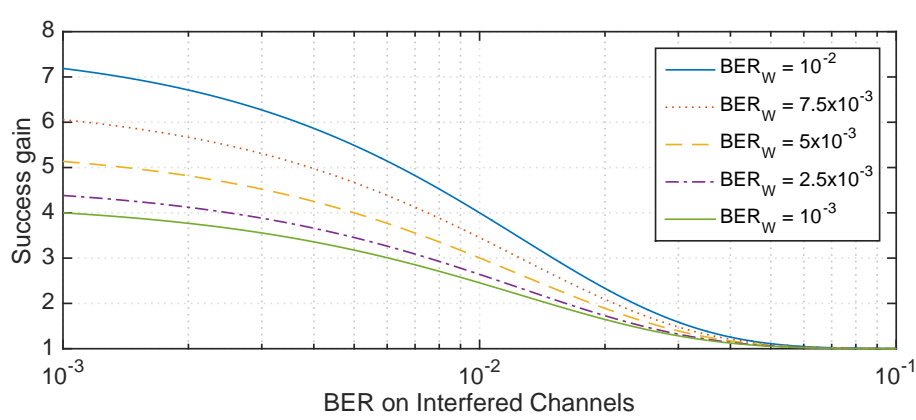

Fig. 2: Values of success gain for different BERs of interfered and white channels.

\begin{tabular}{c|c|c|c|c} 
& Initialization & Weights & Weights & Finalization \\
\hline HS & $(1,2,3,4)$ & $(1,2,3,4)$ & $(1,2,3,4)$ & $\left(\mathrm{ch}_{1}^{\mathrm{W}}, \mathrm{ch}_{2}^{\mathrm{W}}, \mathrm{ch}_{2}^{1}, \mathrm{ch}_{1}^{\mathrm{I}}\right)$ \\
\hline$\Delta$ & $(0,0,0,0)$ & $(9,0,2,0)$ & $(9,9,2,2)$ & $(9,9,2,2)$ \\
\hline $\mathcal{R}^{1}$ & $\{1,3\}$ & $\{1,3\}$ & $\{1,3\}$ & $\left\{\mathrm{ch}_{1}^{\mathrm{W}}, \mathrm{ch}_{2}^{\mathrm{I}}\right\}$ \\
\hline $\mathcal{R}^{2}$ & $\{2,4\}$ & $\{2,4\}$ & $\{2,4\}$ & $\left\{\mathrm{ch}_{2}^{\mathrm{W}}, \mathrm{ch}_{1}^{\mathrm{I}}\right\}$ \\
\hline $\mathcal{R}^{3}$ & $\{3,1\}$ & $\{3,1\}$ & $\{3,1\}$ & $\left\{\mathrm{ch}_{2}^{\mathrm{I}}, \mathrm{ch}_{1}^{\mathrm{W}}\right\}$ \\
\hline $\mathcal{R}^{4}$ & $\{4,2\}$ & $\{4,2\}$ & $\{4,2\}$ & $\left\{\mathrm{ch}_{1}^{\mathrm{I}}, \mathrm{ch}_{2}^{\mathrm{W}}\right\}$ \\
\hline
\end{tabular}

Fig. 3: Example of Whitening Algorithm for $N_{\mathrm{CH}}=4, \mathcal{W}=$ $\left\{\operatorname{ch}_{1}^{\mathrm{W}}, \mathrm{ch}_{2}^{\mathrm{W}}\right\}, \mathcal{I}=\left\{\operatorname{ch}_{1}^{\mathrm{I}}, \mathrm{ch}_{2}^{\mathrm{I}}\right\}, \mathrm{SFS}=2, N_{\mathrm{D}}=2$.

Finally, in Fig. 2 we plot the gain provided combining Eqs. (11) and (13) with varying BER. The results show us that there is a potential gain when the BER on interfered channels is smaller than $10^{-1}$. It is important to note that the gain depends on the probability of success of the white channels, thus, for instance, if external interference affects the white channels, the gain will increase.

\section{B. Whitening Hopping Sequence Design}

Whitening is the reliable hopping sequence design where white channels are fairly provisioned over consecutive transmission opportunities within the deadline. We define reliability as the percentage of application packets delivered within the application's deadline. Our system model assumes a cross layer information exchange between the application and MAC layer. In this way, it is possible to design a reliable hopping sequence with respect to the application's requirements. Furthermore, we define the number of transmission opportunities $N_{\mathrm{D}}$, the amount of TSCH resource blocks available within the deadline. In our previous work [12] we have shown that, in order to guarantee a fair allocation of white channels, each combination of transmission opportunities from the hopping sequence should have the same amount of white channels.

We assume that every mote has exactly one resource block in every Slot-Frame. In this way, there will be exactly $N_{\mathrm{CH}}$ sets $R^{i}, i=\left\{1,2, \ldots, N_{\mathrm{CH}}\right\}$ representing all the possible resource allocations in the Slot-Frame. Furthermore, we can simplify the indexing of the hopping sequence from Eq. (6) to HS $\left(\left(j \cdot \mathrm{SFS}+\mathrm{of}_{i}\right) \bmod N_{\mathrm{CH}}\right)(15)$. Thus, every new transmission opportunity will select a channel from a SFS-shifted version of the hopping sequence.

The whitening algorithm Alg. 1 distributes white channels in the hopping sequence in order to guarantee fairness among different resource allocations. A resource allocation $R^{i}$ is a set of transmission opportunities towards the deadline, in which channel occurrences are mapped to placeholders drawn form the hopping sequence $\mathrm{HS}=\left(1,2, \ldots, N_{\mathrm{CH}}\right)$. A placeholder is a number that corresponds to an available place for a channel. Whitening removes the effect of initial channel selection for a single packet, as it guarantees the presence of a white channel over the different transmission opportunities of a resource allocation.

The sets of resource allocations are generated during the Initialization phase. For every channel offset of ${ }_{i}$ in the hopping sequence, a set $R^{i}$ of $N_{\mathrm{D}}$ placeholders is drawn from the hopping sequence via Eq. (15).

In the Weights phase, the whitening algorithm associates to every placeholder in HS a weight $\delta_{i}$, initially set to zero and contained in the set $\Delta$. The algorithm allocates white channels to placeholders selecting the placeholder $p$ from HS with minimum corresponding weight. At every step, a white channel is allocated to $p$, and $p$ is added to the set $\mathcal{P}$ containing allocated white channels. Then, for every set of resource allocation $R^{i}$ containing $p$, we increment the weights of the non-white placeholders by $\delta_{\min }$. Finally, the weight of the placeholder $\delta_{p}$ is set to $\delta_{\max }+1$, where $\delta_{\max }=N_{\mathrm{CH}} N_{\mathrm{W}} \delta_{\min }$, the maximum number of increments of non-white placeholders during the Weights phase.

The Finalization phase of the algorithm allocates white and interfered channels to the whitening hopping sequence. We cluster all channels into two disjoint sets of white $\mathcal{W}$ and interfered $\mathcal{I}$ channels using Eq. (10). The sets $\mathcal{W}$ and $\mathcal{I}$ contain the list of white and interfered channels respectively, clustered using Eq. (10). For every placeholder in the hopping sequence, a channel is allocated based on its weight. If the weight is bigger than $\delta_{\max }$, the placeholder is substituted with a random white channel from $\mathcal{W}$, otherwise, with a random interfered channel from $\mathcal{I}$.

In Fig. 3, an exemplary run of the whitening algorithm is shown for the scenario with $N_{\mathrm{CH}}=4, \mathcal{W}=\left\{\mathrm{ch}_{1}^{\mathrm{W}}, \mathrm{ch}_{2}^{\mathrm{W}}\right\}$, $\mathcal{I}=\left\{\operatorname{ch}_{1}^{\mathrm{I}}, \mathrm{ch}_{2}^{\mathrm{I}}\right\}, \mathrm{SFS}=2, N_{\mathrm{D}}=2$. During the Initialization phase, the sets $\mathcal{R}^{i}$ are created from the hopping sequence with respect to Eq. (15), and all the placeholders' weights are set to zero. For every white channel, the Weight phase associates a white channel to the placeholder with minimum weight, in this example to the placeholder 1 . Then, it sets its weight to $\delta_{1}=$ $\delta_{\max }+1=9$. Afterwards, it increments $\delta_{3}$ to 2 , as placeholder 3 is included in two sets containing the white placeholder 1. Finally, the placeholders in HS with corresponding weight bigger than $\delta_{\max }$, namely $p=1,2$, are replaced with a random channel from $\mathcal{W}$, and the others $(p=3,4)$ with a random channel from $\mathcal{I}$.

\section{EXPERIMENTAL RESULTS}

In this Section, we present the results of our analysis by means of simulations and measurements. In both cases, we consider a star-topology network. Our results are shown in terms of application layer reliability, i.e., the percentage of application packets delivered within the deadline, and 


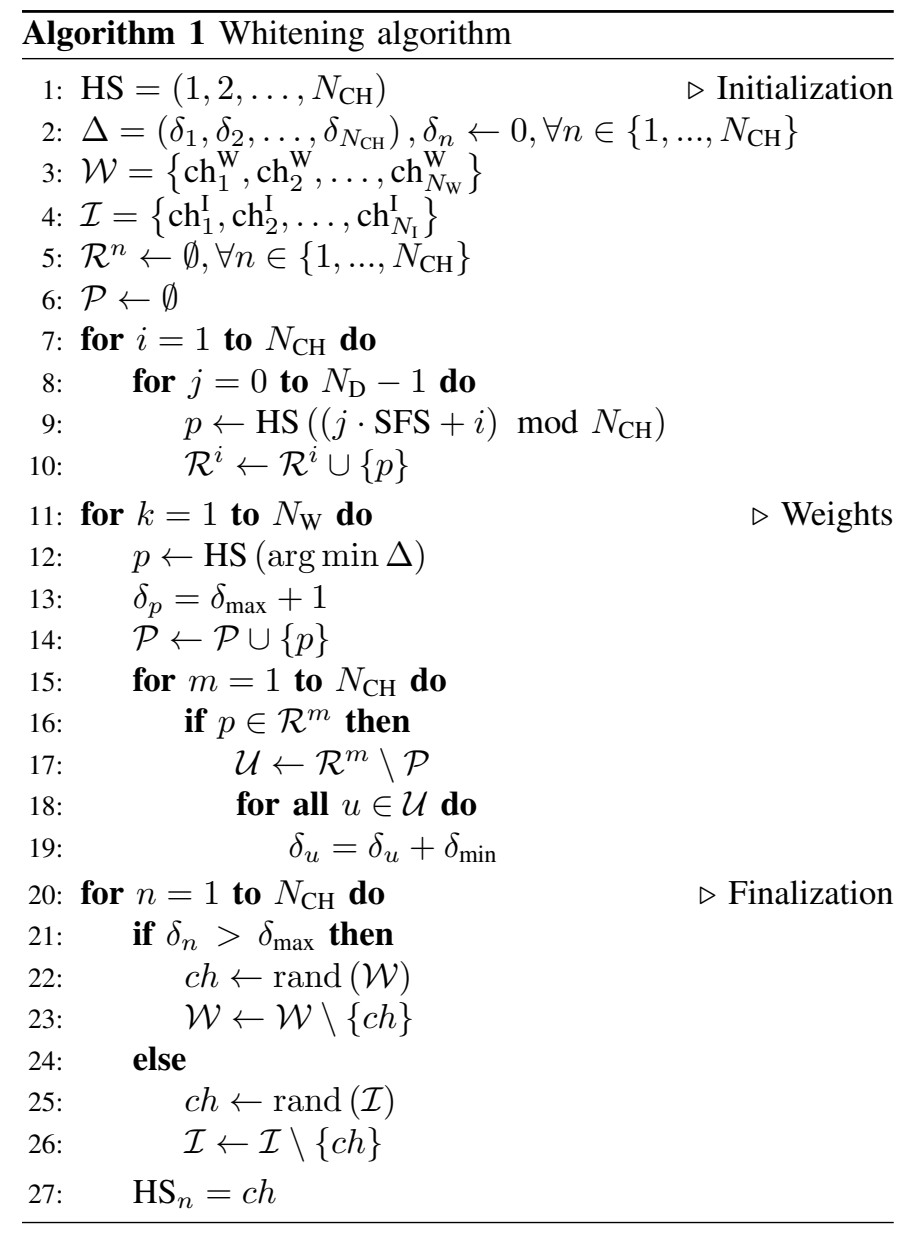

three different hopping sequence approaches: Random (R), whitelisting (WL) and whitening (WG).

Due to the time-varying nature of the real world wireless propagation, we make use of simulations to provide detailed insights in a controlled environment, where different hopping strategies can be compared simultaneously.

On the other hand, we also want to show to the reader that our techniques can be applied to real environments, where Zolertia Z1 devices running OpenWSN can effectively coexists with several Wi-Fi APs.

\section{A. Measurements}

We conduct the measurements to obtain an estimate of packet drop probabilities in a realistic environment and to confirm the gain as presented in Sec. III-A. The measurements were performed on a testbed with 32 motes (16 senders and 16 receivers), and $3 \mathrm{Wi}-\mathrm{Fi} \mathrm{APs}$, in an isolated room with no uncontrolled interference. Every sender generates a burst of 11 packets with $0.5 \mathrm{~s}$ inter-packet interval, and $5 \mathrm{~s}$ interval between the bursts. APs have one client each and receive uplink UDP packets with maximum possible data rate.

Fig. 4 presents measured packet drop probabilities and application layer reliability. From Fig. 4a, we observe that Random hopping sequence outperforms whitelisting given the same number of motes served. This result is straightforwardly

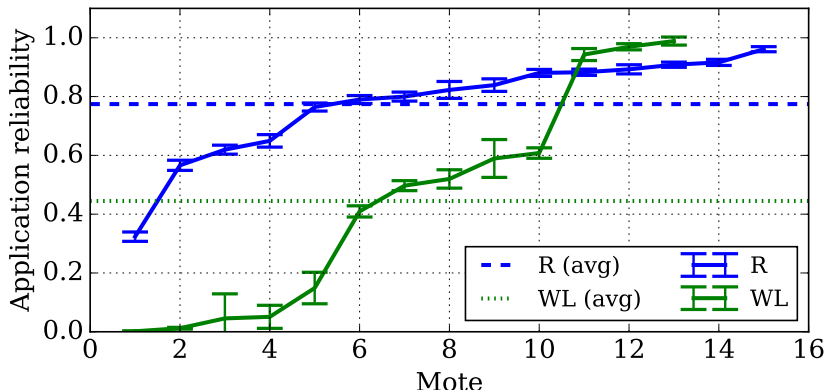

(a)

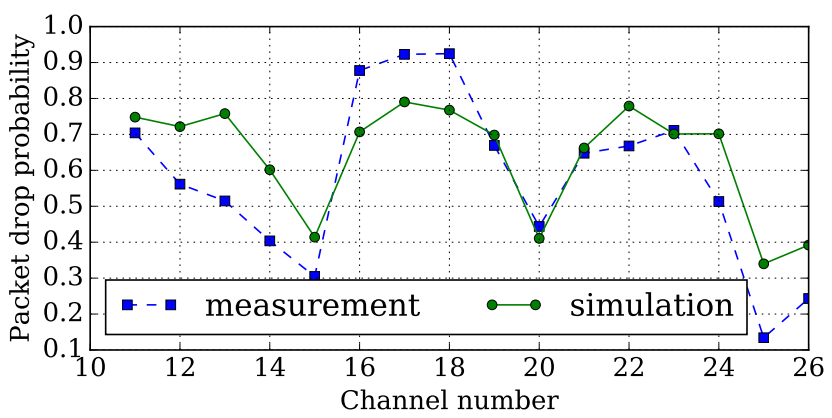

(b)

Fig. 4: Measurements results: (a) sorted per-mote application layer reliability, comparison of Random and whitelist hopping sequence; (b) per-channel link layer reliability, benchmarking the simulation results with measurements.

explained by the fact that whitelisting allows only one transmission attempt (with 4 channels in parallel), whereas Random is allowing four transmissions (with 16 channels in parallel). These results illustrate the gain explained in Sec. III-A.

Fig. $4 \mathrm{~b}$ is a benchmark for simulated packet drop probabilities against measured. We observe that packet drop probabilities for simulation and measurement follow similar dynamics, with channels 15,20, 25, 26 being little affected by interference, and remaining channels highly affected by it. The amplitudes of the packet drop probabilities however deviate from the measurement for certain cases. This could be explained by the multi-path propagation effects not captured in the model.

From the simulated channel drop probabilities we calculate $\alpha$ to fulfill Eq. (10). In particular, we select $\alpha=1.47$ to separate the 4 white channels from the 12 interfering channels.

\section{B. Simulation Schedules}

In order to perform a fair comparison of the different hopping techniques, we present in this section the deadlinebased TSCH schedules used in our simulations.

Schedules for whitelisting and Random methods do not change for different SFSs. However, the whitening schedules are calculated for every SFS according to Alg. 1, in order to synchronize the channels of the hopping sequence with the Slot-Frame periodicity.

Every proposed schedule allocates 16 WSN motes $\left\{\mathrm{m}_{1}, \mathrm{~m}_{2}, \ldots, \mathrm{m}_{16}\right\}$ over the 16 WSN frequencies 


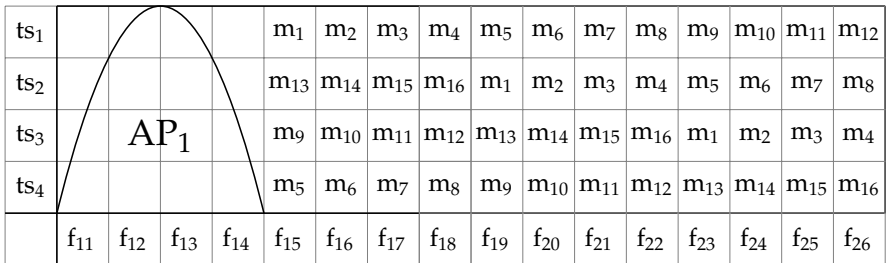

(a) $1 \mathrm{AP}$

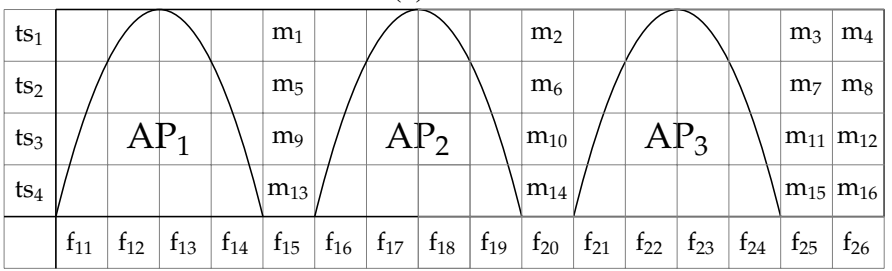

(c) 3 APs

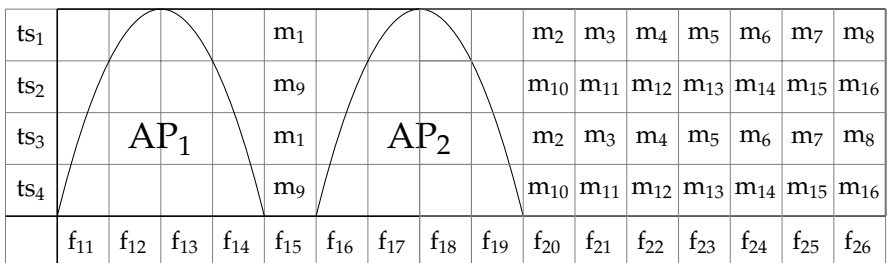

(b) 2 APs

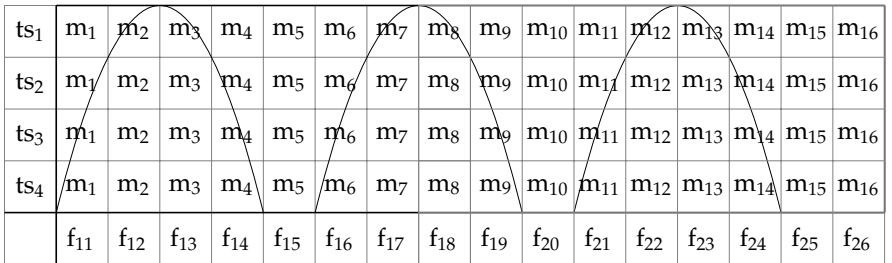

(d) Random

Fig. 5: Exemplary whitelist schedules in presence of interfering Wi-Fi APs (a,b,c), and Random schedule (d).

$\left\{\mathrm{f}_{11}, \mathrm{f}_{12}, \ldots, \mathrm{f}_{26}\right\}$ of the $2.4 \mathrm{GHz}$ ISM band, and a time window of $N_{\mathrm{D}}=4$ time slots $\left(\mathrm{ts}_{1}, \mathrm{ts}_{2}, \mathrm{ts}_{3}, \mathrm{ts}_{4}\right)$ representing possible transmission opportunities before the application deadline. Note that for whitelisting, the actual number of transmission opportunities per individual mote depends on the amount of available whitelisted channels, and, hence, on the number of APs.

Figures 5a, 5b and 5c show the simulated schedules for whitelisting. Fig. 5a shows that, in the presence of one interfering AP on Wi-Fi channel 1, every mote has three transmission opportunities to deliver an application packet. Fig. 5 b shows that the schedule with 2 APs on Wi-Fi channels 1, 6 accommodates two transmissions for every mote. Finally, Fig. 5c shows that, when three interfering APs are active on Wi-Fi channels 1, 6, 11, whitelisting needs all the four time slots to provide a single channel access to a mote.

On the other hand, Fig. 5d shows the exemplary Random schedule where all the channels are used. This schedule neglects the presence of the interfering Access Points and always accommodates four transmissions opportunities for every application packet.

\section{Simulation Results}

Every simulation scenario defines specific values of SFS, transmission power and number of Wi-Fi APs, and consists of 16 transmitting WSN motes. The distance between the transmitters and receivers is fixed to $20 \mathrm{~m}$, and the transmission power is always set to $1 \mathrm{~mW}$. The application running on each transmitter generates packets periodically, with a period that guarantees buffer stability in presence of re-transmissions.

At the same time, Random, whitelist and whitening are simulated in the same wireless environment. For every ASN, wireless realizations for the WSN channels and the Wi-Fi APs are generated and shared among the transmissions of the three schedules. This guarantees the same probability of success for TSCH channels across different schedules for every time slot, and enables comparison.

As shown in Fig. 2, the magnitude of the gain strongly depends on the interference level of the ISM band, thus, in the following simulations we present the effect of interference on reliability and latency.

Fig. 6a shows the behavior of the application drop-rate for different Slot-Frame-Sizes in presence of three APs active on Wi-Fi channels $1,6,11$, with different interference levels: $10 \mathrm{~mW}(\mathrm{~L}), 100 \mathrm{~mW}(\mathrm{M}), 1 \mathrm{~W}(\mathrm{H})$ and $10 \mathrm{~W}(\mathrm{U})$. We can notice that, although the interference level is very high, accommodating re-transmissions on interfered channels provides a considerable reliability improvement with respect of whitelisting. The lower the interference, the higher the gain.

Similarly, Fig. 6b shows the same metric when one, two or three APs are active on Wi-Fi channels 1, 6, 11, with transmission power $1 \mathrm{~W}$. It shows how different ratios of white and interfered channels impact the success gain. As we can see, whitening outperforms whitelisting in every scenario, while Random performs better for 2 and 3 APs.

In both Figures, the oscillating behavior of the Random schedule is explained due to synchronization of the white channels in the transmission opportunities for different SFSs. For instance, for SFS $=4$ the Random hopping sequence is a particular whitening sequence, and, in this case, whitening and Random achieve the same results. However, as shown in Fig. 7a, for SFS $=5$ an initial channel selection can allocate 3 consecutive white channels to some motes, and allocate successive interfered channels to others. Both whitelisting and Random are penalized by the hopping sequence synchronization with the SFS. However, this effect is not present in whitening that has a hopping sequence designed for every SFS. This underlines the importance of the hopping sequence design in presence of interference and application requirements.

Finally, Fig. 7b shows latency values for odd values of Slot-Frame-Sizes when the transmission power is $1 \mathrm{~W}$ and 3 APs are active. Whitelisting has a latency advantage of having one transmission opportunity across all SFSs, while, for other hopping sequences, re-transmissions introduce latency overhead. This is a typical behavior that shows the trade-off between latency and reliability, as shown in [12]. However, whitelisting latencies for SFS $=1$ are higher than Random or 


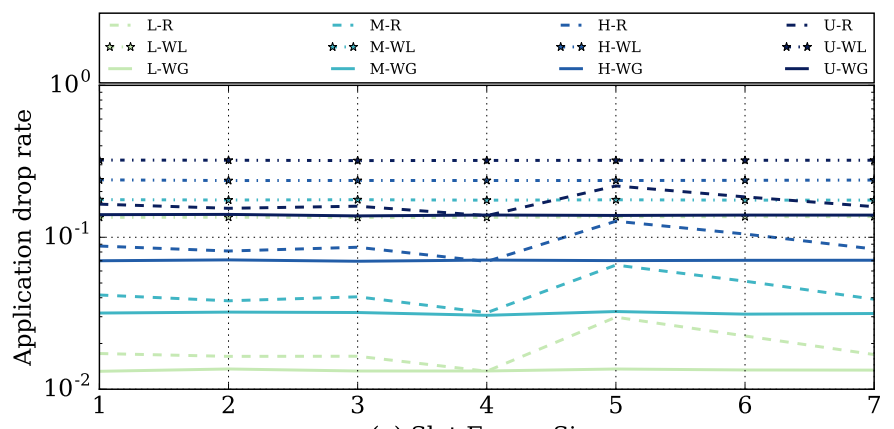

(a) Slot-Frame-Size

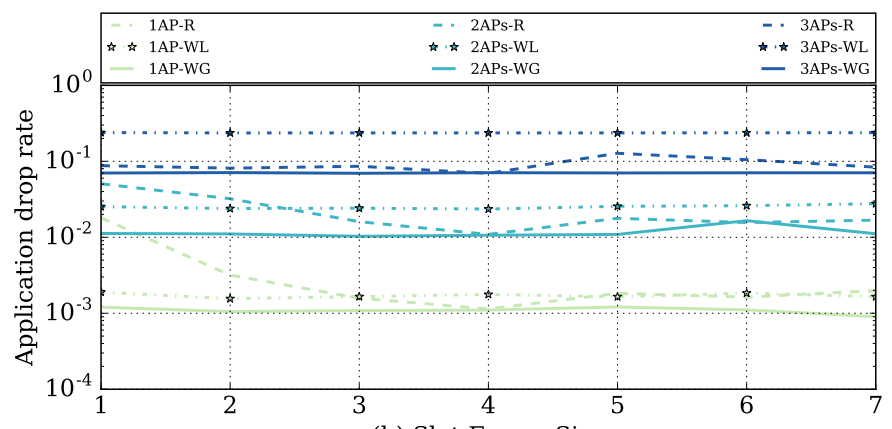

(b) Slot-Frame-Size

Fig. 6: Application reliability for different SFSs for Random (R), whitelisting (WL) and whitening (WG) techniques in presence of different level of interference (a), and different number of APs (b).

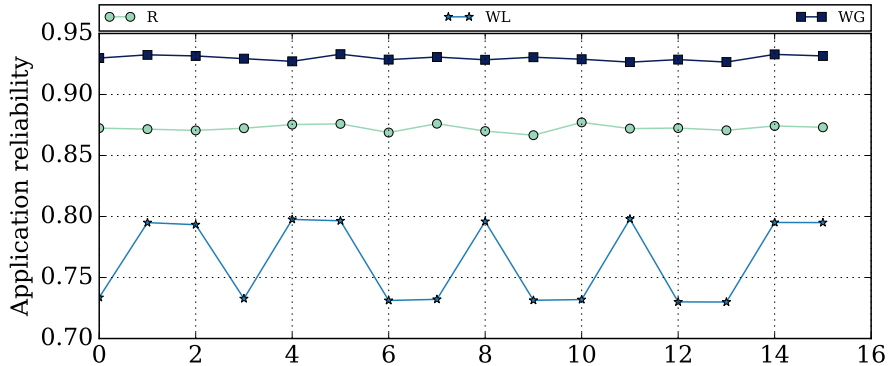

(a) Mote ID

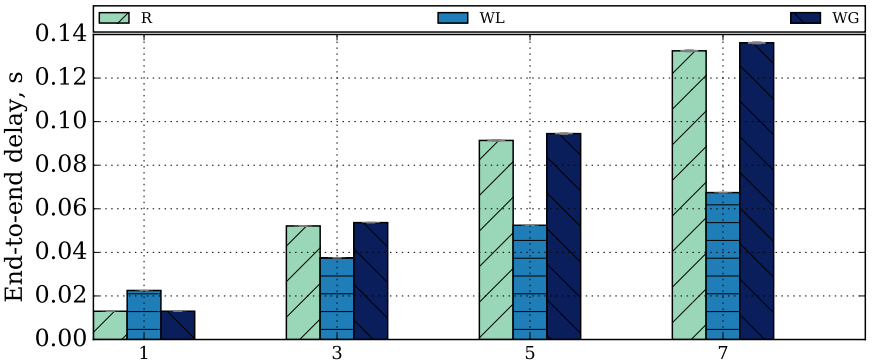

(b) Slot-Frame-Size

Fig. 7: Application reliability for every Mote ID when SFS $=5$ (a), latency comparison per data set vs SFS (b).

whitening latencies due to the longer waiting times between transmission opportunities.

\section{CONCLUSIONS AND FURTHER WORK}

In this paper, we modeled the probability of success of $\mathrm{TSCH}$ resources, and evaluated the gains of using interfered channels together with white channels for transmission. Our simulations and measurements show that, thanks to this gain, the proposed whitening-based hopping sequences outperform whitelisting for deadline-oriented applications in interference conditions. Furthermore, we study the effect of Slot-FrameSize on the hopping sequence, and we show that the whitening hopping sequence design always provides improved reliability. Our results represent a starting point for future work in different interference scenarios, network topologies, and resourceblock-based medium access schemes, e.g., cellular networks.

\section{REFERENCES}

[1] R. Zhang, M. Wang, L. X. Cai, Z. Zheng, X. Shen, and L. L. Xie, "Lteunlicensed: the future of spectrum aggregation for cellular networks," IEEE Wireless Communications, vol. 22, pp. 150-159, June 2015.

[2] H. M. Gürsu, M. Vilgelm, W. Kellerer, and E. Fazli, "A wireless technology assessment for reliable communication in aircraft," in Wireless for Space and Extreme Environments (WiSEE), 2015 IEEE International Conference on, pp. 1-6, Dec 2015.

[3] A. Conti, D. Dardari, G. Pasolini, and O. Andrisano, "Bluetooth and IEEE 802.11 b coexistence: analytical performance evaluation in fading channels," IEEE Journal on Selected Areas in Communications, vol. 21, no. 2, pp. 259-269, 2003.

[4] T. Watteyne, A. Mehta, and K. Pister, "Reliability through frequency diversity: why channel hopping makes sense," in Proceedings of the 6th ACM symposium on Performance evaluation of wireless ad hoc, sensor, and ubiquitous networks, pp. 116-123, ACM, 2009.
[5] "IEEE Standard for Local and metropolitan area networks-Part 15.4: Low-Rate Wireless Personal Area Networks (LR-WPANs) Amendment 1: MAC sublayer," IEEE Std 802.15.4e, pp. 1-225, April 2012.

[6] A. Gonga, O. Landsiedel, P. Soldati, and M. Johansson, "Revisiting multi-channel communication to mitigate interference and link dynamics in wireless sensor networks," in Distributed Computing in Sensor Systems, IEEE International Conference on, pp. 186-193, IEEE, 2012.

[7] M. Vilgelm, M. Gürsu, S. Zoppi, and W. Kellerer, "Time slotted channel hopping for smart metering: Measurements and analysis of medium access," in IEEE SmartGridComm, Nov 2016.

[8] P. Du and G. Roussos, "Adaptive time slotted channel hopping for wireless sensor networks," in Computer Science and Electronic Engineering Conference (CEEC), 2012 4th, pp. 29-34, IEEE, 2012.

[9] R. Tavakoli, M. Nabi, T. Basten, and K. Goossens, "Enhanced timeslotted channel hopping in wsns using non-intrusive channel-quality estimation," in Mobile Ad Hoc and Sensor Systems (MASS), 2015 IEEE 12th International Conference on, pp. 217-225, IEEE, 2015.

[10] C.-F. Shih, A. E. Xhafa, and J. Zhou, "Practical frequency hopping sequence design for interference avoidance in 802.15 . 4e tsch networks," in IEEE ICC, pp. 6494-6499, IEEE, 2015.

[11] R. Soua and P. Minet, "Multichannel assignment protocols in wireless sensor networks: A comprehensive survey," Pervasive and Mobile Computing, vol. 16, pp. 2-21, 2015.

[12] M. Gürsu, M. Vilgelm, S. Zoppi, and W. Kellerer, "Reliable co-existence of 802.15.4e TSCH-based WSN and Wi-Fi in an aircraft cabin," in IEEE Int. Conf. on Communications Workshops, pp. 663-668, May 2016.

[13] I. F. Akyildiz and M. C. Vuran, Wireless Sensor Networks, vol. 4. John Wiley \& Sons, 2010.

[14] M. Z. Zamalloa and B. Krishnamachari, "An Analysis of Unreliability and Asymmetry in Low-Power Wireless Links," ACM Transactions on Sensor Networks (TOSN), vol. 3, no. 2, p. 7, 2007. 